\title{
La présence graduelle du sujet autobiographique : les multiples je de Raymond Depardon
}

\author{
Camille Bui*
}

Resumo: A partir do caso de Raymond Depardon, este trabalho propõe pensar a autobiografia, não como um gênero documentário específico, mas como um impulso afectando gradualmente diferentes tipos de produção. Jornalista e, depois, artista, Depardon afirma cada vez mais o seu «eu» autoral, mas sem nunca perder de vista o desejo de falar sobre o estado do mundo em que vivemos.

Palavras-chave: documentário; autobiografia; Depardon; fotografia; intersubjetividade.

Resumen: A partir del caso de Raymond Depardon, este trabajo se propone pensar sobre la autobiografía, no como un género documental específico, sino como un impulso que afecta gradualmente a diferentes tipos de producción. Periodista priemro y luego artista, Depardon afirma cada vez más su «yo» de autor, sin perder aun así de vista el deseo de hablar de un estado del mundo en el que vivimos.

Palabras clave: documental; autobiografía; Depardon; fotografía; intersubjetividad.

\begin{abstract}
Studying the case of Raymond Depardon, this paper reflects on the autobiography, not taken as a specific documentary genre, but rather as a gradual impulse at work in different types of production. As a journalist and then as an artist, Depardon progressively asserts his authoral first-person without ever losing sight of the desire to speak about the world we live in.
\end{abstract}

Keywords: documentary; autobiography; Depardon; photography; intersubjectivity.

Résumé: À partir du cas de Raymond Depardon, cet article propose de réfléchir à l'autobiographie, non comme un genre documentaire spécifique, mais comme une impulsion à l'œuvre de manière graduelle dans différents types de production. Journaliste puis plasticien, Depardon affirme progressivement son « je »d'auteur, sans jamais perdre de vue le désir de parler d'un état du monde commun.

Mots-clés: documentaire; autobiographie; Depardon; photographie; intersubjectivité.

\footnotetext{
* Université Paris Diderot, Sorbonne Paris Cité, Centre d'Études et de Recherches Interdisciplinaires en Lettres, Arts, Cinéma - CERILAC. 75013, Paris, France. E-mail : camille.bui @univ-paris-diderot.fr

Submission de l'article : 31 décembre 2015. Notification d'acceptation : 13 février 2016.
}

Doc On-line, n. 19, março de 2016, www.doc.ubi.pt, pp. 22-41. 


\section{Souci du monde et récit de soi}

D'un point de vue pragmatique, le cinéma documentaire correspond à un mode d'adresse particulier au spectateur : Jean-Luc Lioult, reprenant les distinctions faites par Jean-Marie Schaeffer dans son ouvrage Pourquoi la fiction? (1999), propose de parler du documentaire en tant qu' " assertion sérieuse consentie », soit un mode communicationnel qui contraste avec la « feintise ludique partagée » caractéristique de la fiction (Lioult, 2004 : 33). Le film documentaire est une assertion sérieuse au sens où les auteurs affirment quelque chose, " déclarent que quelque chose existe, s'est produit » et attendent des spectateurs qu'ils « reçoivent leur déclaration comme sincère et véritable sur un état du monde » (Lioult, 2010). L'assertion est « consentie » lorsque s'établit un consensus entre producteurs et récepteurs du discours, un pacte de lecture autour de la nature documentaire du film.

Cette définition pourrait s'appliquer à tout type d'image documentaire, mais elle fait implicitement référence à une tradition cinématographique concernée par l'« état du monde ». En effet, depuis ses débuts, l'ambition documentaire au cinéma correspond non seulement à un mode d'adresse mais aussi à un esprit spécifique. Pour ceux qui s'inscrivent dans cette filiation, faire des films documentaires est un projet esthétique autant que métaphysique et politique : il s'agit de rendre compte, d'interroger voire de transformer le monde commun, en élaborant du sens partageable. Avant les années 1960, dans la rencontre entre l'auteur et le «monde », l'accent était mis sur le second. Figure fondatrice, Robert Flaherty fût ainsi très déçu par la première version de son film tourné dans le Grand Nord canadien lorsqu'il se rendit compte que ses spectateurs s'intéressaient plus à son expérience d'explorateur qu'au mode de vie du peuple qu'il avait filmé (Calder-Marshall, 1963 : 76). Si, par la suite, il collabora avec ses personnages pour réaliser Nanouk (1922), ce n'était pas pour faire le récit de son aventure parmi les Esquimaux, mais plutôt pour s'assurer que son œuvre serait au service de la réalité de la communauté : lui devait s'effacer derrière sa caméra. Cette anecdote inaugurale permet de revenir sur la place classique du documentariste : témoin s'élançant au-devant du monde et des autres, il existerait en tant que relais, médiateur et non comme sujet en soi. Dès ses débuts, le documentaire est assumé comme mise en scène cinématographique du réel, mais il n'est pas question pour son auteur d'y apparaître autrement qu'à travers son regard sur le monde.

Dès les années 1960 et la naissance de la modernité documentaire (PierronMoinel, 2010), de nombreux cinéastes remettent en cause ce paradigme de l'auteur transparent et/ou omniscient, tant d'un point de vue artistique qu'idéologique. Émergent alors des formes de cinéma direct réflexif dont Chronique 
d'un été (1961) de Jean Rouch et Edgar Morin est l'un des plus fameux jalons. Les deux auteurs de ce film-manifeste ne se prennent pas eux-mêmes comme sujets, mais ils ne se retranchent plus derrière la caméra. Ils se mettent en scène comme prenant part à l'expérience sociale du tournage aux côtés de leurs personnages, et livrent aux spectateurs les clés de leur démarche aussi bien que leurs doutes et interrogations. Le devenir-personnage du documentariste à l'œuvre dans le mode participatif du documentaire prend une forme radicale dans le genre autobiographique. Le cinéaste est alors complètement localisé « sur la scène » (Nichols, 2001 : 116) : il prend corps. Cette apparition progressive du « je » dans la tradition documentaire se traduit aujourd'hui par l'importance des films à la première personne réalisés par des cinéastes comme Ed Pincus, Ross McElwee, Jonathan Caouette, Carmen Castillo, Edgardo Cozarinsky, ou encore Dominique Cabrera, Alain Cavalier et Agnès Varda. Mais, plus qu'une rupture avec les ambitions classiques du documentaire, ce tournant révèle la part subjective qui est à l'œuvre dans tout film, même si elle l'est parfois de manière refoulée.

Pour réfléchir à cette "gradation autobiographique », l'œuvre du photographe et cinéaste Raymond Depardon est particulièrement intéressante. Celui-ci est d'abord reconnu pour son travail photographique dans les années 1960 : grand reporter opérant sous le régime de l'objectivité journalistique, il est alors censé demeurer extérieur à son sujet. Dès les années 1970, débute en parallèle son expérience de cinéaste. Toujours en prise avec les grands événements du monde, Depardon est amené à s'intéresser au réel dans ses temps forts : guerres, actualités politiques ou sportives, apparitions publiques de célébrités sont ses sujets quotidiens. Assez rapidement, il conquiert son indépendance par rapport aux commandes et aux critères journalistiques, pour finir par s'extraire complètement du système de la presse. Depuis les années 1980, il se rapproche du milieu artistique et affirme de plus en plus sa subjectivité, jusqu'à réaliser des œuvres explicitement autobiographiques.

L'évolution de la démarche de Depardon est ainsi marquée par des variations du je autour d'une même aspiration à documenter le réel. Son œuvre voit se succéder ou se combiner différents modes d'implication de l'auteur dans les images, et doit beaucoup à la diversité de ses influences qui vont du reportage d'actualité au cinéma direct (Leacock puis Wiseman), de la photographie de l' «instant décisif » (Cartier-Bresson) au documentaire social américain (Klein, Frank). L'analyse de sa trajectoire, en photographie et au cinéma, permet de se pencher sur les enjeux théoriques et historiques de l'émergence graduelle du je au sein de la tradition documentaire. Autrement dit, à travers le cas de Raymond Depardon, il s'agit plus généralement d'interroger la reconfiguration des 
liens entre l'auteur, le monde, les images et le spectateur qu'opère l'impulsion autobiographique au sein même du désir de «parler d'un état du monde ».

Le corpus documentaire dans lequel s'inscrit Depardon se distingue d'autres œuvres filmiques à la première personne qui privilégient un mouvement de subjectivation, parfois jusqu'à brouiller toute référence indicielle au réel filmé. Si les méditations ou journaux visuels de certains cinéastes expérimentaux tels Jonas Mekas, Stan Brakhage ou Hollis Frampton participent à la tendance générale à l'autobiographie dans le cinéma indépendant, ceux-ci dialoguent plus directement avec l'histoire des arts plastiques qu'avec la tradition documentaire. Car, pour reprendre les termes de Jim Lane, le documentaire autobiographique se caractérise, lui, par une "tension that hinges on the documentary impulse to objectively record an historical world 'out there' and on the autobiographical impulse to subjectively record a private world 'in here' » (Lane, 2002 : 4). Cette tension entre un mouvement centripète (aller au-devant du monde) et un mouvement centrifuge (revenir sur soi) n'est pas seulement esthétique, elle est aussi éthique. En effet, l'émergence du je de l'auteur comme forme extrême de la réflexivité procède d'une critique de l'énonciation classique et de ses ressorts idéologiques qui transforme les manières de dire la vérité du monde.

\section{Désir d'objectivité et refoulement du je}

La décennie 1960 constitue une période florissante pour les agences de presse, à la fin de l'âge d'or du photoreportage dont le début coïncide avec la guerre d'Espagne entre 1936 et 1939. Du côté des arts plastiques, dans les années 1960, la photographie est à la fois outil et médium de l'art contemporain pour les artistes de la remise en cause de la modernité. C'est dans ce contexte historique que Raymond Depardon commence sa carrière au sein de la photographie de presse. Il travaille comme reporter dans les années 1960 au sein de l'agence Dalmas, à Paris. En tant que pigiste puis employé permanent, il est envoyé sur le terrain pour couvrir différents événements. Les premiers travaux photographiques de Depardon sont marqués par ce qu'André Rouillé décrit comme la morale du reportage classique, selon lui « l'expression la plus avancée de la conception selon laquelle l'image est la reproduction d'une chose qui lui serait antérieure, d'un dehors, d'un original qui lui préexisterait» (Rouillé, 2005 : 89). Chargé de rapporter des informations en se déplaçant sur le terrain, le reporter ne doit pas se laisser aller à charger les images de son interprétation subjective. Ses photographies d'actualité politique, sportive ou « people» sont transmises à un public à qui l'on veut montrer la réalité des faits : elles doivent 
donner à voir ce qui s'est effectivement produit, et pour cela s'effacer en tant que médiation, instance de représentation.

L'image de presse vaut en effet pour son contenu référentiel : les scandales que certaines photos ont pu déclencher sont la preuve de leur force indicielle (« ça-a-été », Barthes, 1980). Depardon est envoyé par l'agence Dalmas dans diverses missions dont la première fut «SOS-Sahara » en août 1960, dans l'Algérie colonisée. Au cours de cette expédition, Depardon fut confronté à une situation dramatique qui devint un événement médiatique. Des soldats s'étaient perdus dans le désert, et l'équipe de Depardon retrouva plusieurs d'entre eux, permettant leur sauvetage alors qu'ils se trouvaient à un stade avancé de déshydratation. Depardon en tira des clichés dont l'un fera la couverture du magazine Paris-Match. Depardon raconte qu'un militaire haut placé avait voulu étouffer l'affaire et lui avait demandé de livrer ses films, ce que ce dernier réussit à éviter grâce à un mensonge. C'est ainsi que l'information fut diffusée, et la vérité des événements révélée au monde par le biais de la presse : Depardon explique qu'il apprit «plus tard, que ce gradé avait annoncé aux familles que [les soldats] étaient morts au combat » (Depardon, 2007 : 28). Cet exemple, fondateur de la carrière de Depardon-reporter, montre à l'œuvre la capacité de la photographie sur laquelle repose le photojournalisme classique de révéler au monde des événements marquants. Cette mission lui permit d'abandonner le simple statut de pigiste et d'être promu au sein de l'agence en tant que photoreporter capable de couvrir des événements importants du « Viêt Nam à Moscou en passant par l'Afrique ». Cependant, cette reconnaissance ne concernait pas d'abord la singularité de son regard en tant qu'auteur : la valeur de ses images reposait avant tout sur leur puissance référentielle.

Le travail du photographe se réduisait alors à « trouver la bonne place » (Depardon, 2007 : 29), à être là au bon moment afin de trouver le bon point de vue : celui qui servirait le sujet de façon optimale. Mais dans cette recherche de la «bonne rencontre avec le réel » se dessine a minima l'implication du sujetauteur dans la production des images. Le photographe adopte la position du « chasseur » à la fois soumis à un réel qu'il ne maitrise pas et prêt à réagir. Pour réussir son image, il doit déclencher au bon moment afin de concentrer la force d'un événement qui dure en un instant privilégié, « représentatif ». La pratique d'Henri Cartier-Bresson dont s'inspire alors Depardon procède de cette idée selon laquelle le bon cliché est une synthèse instantanée de ce qui s'est déroulé dans le temps. Cartier-Bresson se dévoue à la recherche de ce fameux « instant décisif » et parle de «prendre sur le vif des photos comme des flagrants délits ». Chez lui la posture de chasseur est radicale : «Photographier : c'est dans un même instant et en une fraction de seconde reconnaître un fait et l'organisation 
rigoureuse de formes perçues visuellement qui expriment et signifient ce fait » (Cartier-Bresson, 1952).

Cette recherche de la «bonne rencontre » avec le réel guide les débuts photographiques de Raymond Depardon. Il met cela en œuvre dans ses reportages tels que ceux des Jeux Olympiques de Tokyo en 1964 et de Mexico en 1968. Dans ces derniers, la plupart des images témoignent de moments sportifs forts. Par exemple, une photo nous montre l'arrivée de l'Américain William Mills à la fin du parcours de dix mille mètres dont celui-ci sort médaillé d'or. Une autre nous fait voir la manifestation de joie de l'athlète française Colette Besson, gagnante du « 400 mètres en 52 secondes ». D'autres images apparaissent comme des moments d'action moins forts mais rendent compte de la préparation du temps clef qui les suit immédiatement. Les photographies de sport sont dépendantes de l'événement, que la prise de vue se situe au moment du temps fort ou bien juste avant ou après. Pour transmettre la puissance du réel à son image, le photographe doit faire preuve d'une maîtrise technique : la performance du sportif est doublée de celle de l'opérateur. En creux, la photographie documente l'expérience du photographe, même si celle-ci a pour vocation de s'effacer devant le référent.

De son propre aveu, les premiers films de Depardon « ont été comme un prolongement naturel de la photographie » (Depardon, 2007 : 37). En 1969, il filme, un an après les événements du Printemps de Prague, les funérailles de Ian Palach. Cet étudiant tchécoslovaque s'immola par le feu le 16 janvier 1969 pour protester contre l'invasion de l'Union Soviétique. Le court-métrage documentaire Ian Palach est constitué de plans filmés en caméra portée se succédant par un montage cut, sans commentaire, avec un son pris en direct. Les plans sont relativement longs, et tous sont caractérisés par le tremblement dû au filmage à l'épaule, qui laisse transparaître la présence du corps filmant.

Mais ce premier film obéit à une logique proche de celle du photoreportage : à la fois par le choix du sujet (un événement d'actualité important et ponctuel), par la maîtrise du médium orientée vers les qualités du réel que ce dernier est à même d'enregistrer (aspect visuel, temporel, sonore, cinétique), ainsi que par l'adoption d'un point de vue extérieur aux faits. Car la présence derrière la caméra est avant tout celle d'un homme de métier déterminé par son outil d'enregistrement ainsi que par la réalité à capter. Ainsi, Depardon se contente-t-il d'enregistrer les faits et, lorsqu'il interviewe une jeune fille à propos des événements, il ne fait que rapporter ses propos. Le montage est chronologique et narratif, obéissant aux critères classiques de la transparence réaliste, selon lesquels le spectateur ne doit pas se « heurter » à la forme du film. 
Pour son premier long-métrage, dans lequel il retrace la campagne de Valéry Giscard d'Estaing aux élections présidentielles de 1974, Depardon poursuit dans la voie d'un cinéma direct proche du journalisme. Inspiré par Primary (1960) de Robert Drew et Richard Leacock, avec sa caméra légère, Depardon et son ingénieur du son vivent au jour le jour aux côtés du candidat : des coulisses de la vie politique au-devant de la scène, ils sont présents à tous les moments clefs. Le début du film est proche d'un reportage conventionnel, puis, Depardon ayant avisé l'homme politique que la routine n'était pas assez intéressante pour qu'il continue dans ces conditions, il obtient l'autorisation de suivre celui-ci partout. Depardon a alors accès à tous les moments habituellement non médiatisés : il assiste à des réunions stratégiques, aux coulisses du débat télévisé avec l'adversaire François Mitterrand... Il parvient à s'accommoder des contraintes pour mettre en scène le personnage de Giscard d'Estaing au-delà du registre télévisé convenu. Une fois élu, le président s'opposera d'ailleurs à la diffusion du film, le trouvant trop peu flatteur à son égard : 1974, une partie de campagne ne sortira qu'en février 2002.

Pour ce film, Depardon a pourtant d'abord été guidé par la recherche des temps forts de la campagne : meetings, réunions, interventions télévisées. Il évoque même ses déceptions durant le tournage lorsque rien ne se passait, qu'il n'y avait rien d'intéressant à filmer. Par exemple, quand Giscard d'Estaing se repeigne pendant un voyage en voiture, l'acte insignifiant lui apparaît d'abord trivial et sans intérêt. Ce n'est qu'au montage que Depardon dit avoir compris l'intérêt de temps faibles qui participent au sens du film : sa démarche spontanée de photographe d'actualité le poussait d'abord à vouloir capter des «actions spectaculaires ou décisives » (Parente, 2005 : 147).

Ainsi, dans ses photos comme dans ses films, le premier Depardon adopte une même démarche : il aspire à documenter une réalité extérieure, à travers la saisie de temps forts. Il évoque la jouissance qui accompagne l'acte photographique, produite par l' «instant décisif »: lorsque le temps fort de la réalité et celui de la photographie coïncident. Ce plaisir d'avoir pu figer ce qui «a été » est lié à la captation de l'événement en des points remarquables, sélectionnés par la photographie. Lorsque Depardon se met à filmer, la «bonne rencontre » s'opère autrement : l'événement s'étire dans le temps. Selon André Parente, le cinéma direct de Depardon-reporter est un cinéma de «l'image-action » deleuzienne. Sa démarche peut être mise en parallèle avec les propos de Leacock rapportés par Parente : «ce qui nous intéresse avant tout, c'est le comportement de certaines personnes impliquées dans le déroulement d'un événement important » (Parente, 2005 : 146). La conception du direct tel que le pratique Depardon serait « un cinéma construit « selon l'optique fondamentale fournie 
par la réalité » (Marsolais, $1974:$ : 1) qui a « l'attitude supposée de saisir le réel sur le vif » et repose sur « le culte d'un réel préexistant, de la réalité brute, saignante, 'telle quelle' » (Parente, 2005 : 146). Dans cette démarche, l'auteur met sa virtuosité au service de ce qu'il filme : le sujet-auteur est volontairement refoulé en hors champ.

Toutefois, dès ses débuts, Depardon s'autorise des pas de côté par rapport aux paradigmes de l'instant décisif et de l'image-action. D'autres éléments précoces de sa carrière témoignent d'une prise de liberté : l'apparition d'un commentaire à la première personne dans les différents reportages au Tchad dans les années 1970, ou l'exercice d'une image moins transparente et plus plastique à quelques reprises. Ainsi dans Tchad 1 : L'Embuscade (1970), plusieurs plans se terminent par des arrêts sur images. On peut voir dans cette intervention une volonté de figer des instants dramatiques afin de donner un meilleur accès photographique aux faits. Mais elle peut aussi être vue comme un geste de distanciation critique faisant apparaître au spectateur l'artificialité du réalisme filmique. Cette proposition singulière permet d'entrevoir un Depardon qui n'adhère pas systématiquement à une conception objectiviste du documentaire.

\section{L'émergence du sujet, entre autobiographie et signature du neutre}

Dès les années 1970, d'autres influences nourrissent la production de Depardon. Ce dernier, dans une interview avec Jean-François Chevrier, évoque lui-même ce changement d'orientation :

À la fin des années 1970, conscient ou inconscient, je romps avec le photojournalisme, je l'exprime dans Notes. Je commence à tourner un certain nombre de films, qui sont sans doute, pour certains, le prolongement de mon photojournalisme et à mon avis pas du tout : c'est au contraire une rupture et même un rejet. Alors, c'est sûr que, comme il y a eu des Américains qui m'ont servi de guides pour la photo - Walker Evans, Robert Frank -, il y en a d'autres qui m'ont servi pour le cinéma - Leacock, Wiseman et les frères Maysles. (...) J'ai cru d'abord que Leacock était plus important que Wiseman, et maintenant je m'aperçois que c'est le contraire. D'abord, les films de Leacock étaient marqués par l'événement, par le reportage, par le personnage, par des situations dramatiques, etc. Alors que Wiseman s'est fixé sur des choses de la vie quotidienne, sur des lieux, sur des unités de lieu, de temps (Depardon, 2006: 85-86).

Depardon s'affilie ainsi progressivement à une pratique auteuriste des images documentaires, en s'inscrivant dans une tradition distincte du reportage, à l'heure où s'amorce une " crise du photojournalisme » liée à plusieurs facteurs. La télévision devient une concurrente importante dans le domaine de 
l'actualité, le photojournalisme devient donc moins rentable. La tâche principale assignée jusqu'alors aux photoreporters était justement de «couvrir» les grands événements du monde, la photographie était alors subordonnée à l'information, elle était l'information elle-même. Or la télévision, par ses moyens techniques et son large public, devient le premier média de transmission rapide des informations provenant du monde entier. De plus, la presse écrite subit les conséquences économiques du choc pétrolier de 1973 qui est à l'origine d'une forte inflation, faisant augmenter les prix du papier ainsi que celui de la poste :

Les prestigieuses revues anglaises et américaines Look et Life disparaissent, privant les photographes de publications possibles, et les agences de photographie d'actualité des années 1950 sombrent : Europress, les Reporters associés, Dalmas et Apis ferment à l'orée des années 1970 (Deligny, 2004).

Dans ce contexte, des agences d'un autre type émergent dont l'agence Gamma, fondée par Raymond Depardon en 1966, puis Sipa (1969) et Sygma (1973). Ces agences commerciales rompent avec le modèle précédent au sein duquel les photographes étaient anonymes et n'avaient pas de droit sur leurs images. Le nouveau système « instaure le partage équitable des frais et des bénéfices entre le photographe et l'agence, et lui accorde la mention de son nom lors des publications et la propriété de ses négatifs » explique Aurore Deligny. Selon cette dernière :

Au cours des années 1970, Paris supplante à nouveau New York en tant que plaque tournante du photojournalisme. Cependant, l'important réside moins dans la recherche d'une esthétique et d'un point de vue original que dans le fait de répondre à un marché spécifique, celui de la presse. Asservies aux impératifs de cette dernière, les agences tendent à surenchérir sur « la rapidité et le spectaculaire », avec pour conséquence un « appauvrissement progressif du contenu $\gg$ du photojournalisme. (Deligny, 2004).

C'est avec ce photojournalisme contemporain subordonné au marché que rompt Raymond Depardon afin de développer son regard d'auteur. Son ouvrage Notes est significatif de cette prise de contrôle du photographe sur son projet. Ce recueil est publié en 1979 par la petite maison d'édition indépendante Arfuyen. Il regroupe des photographies faites par Depardon à Beyrouth puis au Pakistan et en Afghanistan, durant l'hiver 1978. S'il s'agit encore de photographies de combattants, les motivations du voyage sont bien différentes des fois où il est allé couvrir les événements pour le compte de la presse. Cette fois, c'est une raison personnelle et même sentimentale qui guide son projet. L'auteur explique qu'une désillusion amoureuse l'a décidé à quitter Paris, « à partir à la guerre et à écrire un journal pour prouver son désir et son attachement tout au long de ce reportage que personne ne lui avait commandé » (Depardon, $2006: 8$ ). 
Si son voyage personnel l'amène sur des lieux du monde où quelque chose se passe, il aborde les faits et les rencontres dans un autre état d'esprit que celui du chasseur d'images qu'il a pu être, sous l'emprise de la commande. Dans son recueil Notes, les photos ne sont pas en rupture radicale avec le registre du reportage de guerre, mais le livre dans son entier offre un résultat tout à fait différent. Il y retrace le parcours personnel qui le conduit à relier Beyrouth à l'Afghanistan. Le livre se fait ainsi journal intime, mêlant les interrogations du photojournaliste aux états d'âme de l'homme ordinaire. Le voyage journalistique devient du même coup voyage intérieur. Depardon commence ainsi à se réapproprier ses images à travers un projet dont le sujet est ambigu : il photographie encore la guerre et les combattants, mais en y associant son commentaire et son itinéraire subjectif. Le sujet journalistique devient aussi autobiographique.

Notes apparaît comme symptomatique d'un phénomène lié à la crise du photojournalisme qui questionne « la figure héroïque du photoreporter et du mythe de l'événement » et voit surgir « la notion d'auteur, calquée sur le cinéma du même nom » (Roussin, 2006 : 35-36). La remise en cause d'un photojournalisme purement redevable de l'événement devant lequel le photographe s'efface s'incarne également dans la commande de Libération à Depardon, durant l'été 1981. Depuis New York, celui-ci envoie au quotidien une photographie par jour. La rédaction décide alors de soustraire une page de chaque édition à l'actualité. Le photographe français est invité à livrer sa vision de la grande ville américaine. Il est complètement libre : sa seule contrainte est d'envoyer un cliché chaque jour. Chaque image est accompagnée de quelques mots, d'une phrase portant souvent sur les états d'âme de Depardon lui-même. Il s'agit d'une expérimentation journalistique qui permet au photographe de réaliser des images sans commande définie, sans avoir « à raconter une histoire, à couvrir un événement ». Ses images ne sont pas spectaculaires, mais plutôt esthétisantes, montrant la ville à travers un regard singulier, s'arrêtant sur des paysages, des perspectives, des anonymes.

Depardon photographe urbain livré à ses aspirations personnelles revendique des influences américaines plutôt que françaises : il ne cherche pas à s'inscrire dans le réalisme poétique de Robert Doisneau ou d'Henri CartierBresson. Cette photographie humaniste particulièrement reconnue après la Seconde Guerre mondiale envisageait la rue comme le lieu privilégié pour capter une humanité émouvante. Depardon s'intéresse bien plus à la photographie de Robert Frank dont l'ouvrage Les Américains - publié en 1958 - est fondamental dans le renouvellement de la photographie vers une expressivité nouvelle. Ce livre rassemble des photographies prises entre 1955 et 1956 sur la route 
américaine. Pour André Rouillé, Robert Frank place « sa subjectivité au centre de sa démarche » et opère ainsi le passage d'un régime de la «photographiedocument » à un régime de la «photographie-expression ». Ce mouvement correspond également à l'évolution de Depardon :

Le sujet était auparavant l'observateur central, l'opérateur technique, le garant de l'unité esthétique de l'image et de sa fidélité aux lois de la représentation perspectiviste [cf. Cartier-Bresson] (...) Avec Robert Frank, « je » gagne en humanité et en subjectivité. C'est un «je » photographique arrimé de façon pleinement assumée à un vécu personnel, sentimental voire intime. (Rouillé, $2005: 223$ ).

Avec Notes (1979) et Correspondance New Yorkaise (1981), Depardon reste lié au domaine du journalisme : du fait du sujet choisi pour le premier projet et par sa collaboration avec le quotidien Libération pour le second. S'il est libéré des contraintes de l'information d'un photojournalisme «de masse », il reste fidèle à la référentialité propre aux deux médiums photographiques. Sa subjectivité s'affirme par l'introduction explicite du «je » mais aussi dans la recherche obsessionnelle de ce qu'il nomme la «bonne distance » : à mi-chemin entre empiètement et indifférence au territoire de l'autre. Cette éthique, à rebours de l'extériorité supposée du reporter, consiste à rencontrer l'autre - filmé ou photographié - sans se faire juge ou voyeur, dans un délicat mélange d'empathie et de pudeur. Jacques Rancière voit la marque de cette éthique dans la photographie du phalangiste prise à Beyrouth et qui introduit Notes :

Le milicien libanais qui fait corps avec l'horizontalité de son fusil est sans visage. Ce n'est pas seulement qu'il n'eût pas fait bon être en face. C'est que la photographie de Raymond Depardon s'interdit de lire sur le visage d'un tireur les raisons de son énergie meurtrière. Et même devant les détenus de la prison de Kigali, accusés de génocide et, pour nombre d'entre eux sans doute, avec quelques raisons, sa caméra s'interdira de savoir si et pourquoi ils ont participé au massacre. « Je filme seulement des corps, dira-t-il. Il n'y a peut-être rien à comprendre ». L'image ne s'attribue pas le pouvoir de révéler le secret d'un geste, de déchiffrer la pensée qui met le combattant en ce lieu. (...) Ainsi l'image qui semblerait le mieux « parler par elle-même » est-elle soumise à une stratégie déceptive. (Rancière, $2000: \mathrm{np}$ ).

Alors qu'en tant que reporter Depardon entretenait « un rapport direct et réglé avec le réel », il glisse ainsi progressivement vers « un rapport indirect et libre » selon Rancière. Sa pratique photographique remet en cause le mythe de l'objectivité journalistique. Dire « je » par la voix et l'image, c'est gagner en humilité dans la rencontre avec l'autre et déconstruire l'illusion idéologique d'une image vraie qui parlerait naturellement, depuis un lieu abstrait. En effet, l'invisibilisation du sujet-auteur dans le reportage classique participe d'une si- 
tuation d'énonciation liée à une forme de domination symbolique, synthétisée par la formule : « it speaks about them to you» (Nichols, 2001 : 59).

Rompre avec le journalisme pour Depardon va avec une prise de conscience de l'impossibilité d'une image « transparente » et entraine une remise en cause du bon sujet à traiter, du « grand événement » dont la couverture serait dictée par la réalité. Depardon n'abandonne pas le cinéma direct, cependant, il se détache peu à peu de la tendance de la living camera affiliée au groupe de presse Time Inc. Même si son cinéma demeure moins autobiographique que ce qui s'initie dès les années 1980 dans son travail photographique, il existe quelque chose de commun dans sa pratique des deux médiums. L'événement est désormais placé dans un cadre ou dans un temps qui l'excède voire qui le place en hors champ de l'image. À l'opposé du goût pour les grands événements d'une frange du cinéma direct, Parente, reprenant Deleuze, expose l'idée d'un «cinéma indirect libre » dont le cinéma de Jean Rouch serait une des manifestations. Depardon évoque lui-même l'importance de sa rencontre avec Rouch ainsi qu'avec Chris Marker et Fred Wiseman. Ce cinéma indirect libre serait caractérisé par l'abolition du culte du réel préexistant au film, et donc de la croyance en une signification a priori de ce réel. Cette tendance du cinéma direct se trouve coïncider avec le mouvement du cinéma moderne : «Tout le cinéma dit moderne, à commencer par le néoréalisme, témoigne de l'insignifiance des événements. Ce n'est pas tant que les grands événements n'existent plus, mais les personnages ne sont qu'à peine concernés par eux » (Parente, 2005 : 147).

Un des films de Depardon les plus radicaux dans l'abandon de l'événement journalistique est celui qu'il tourne en 1980 dans un hôpital psychiatrique en Italie : San Clemente. Il s'agit d'un asile-hospice où il s'est déjà rendu plusieurs fois pour réaliser des photographies, et à propos duquel il dit : "Pas de scoop pas de princesse, je suis déconcerté, je ne sais pas quoi photographier ». Cette fois, il tourne avec Sophie Ristelhueber qui effectue la prise de son en direct, et réalise un long-métrage. Il s'agit d'un film en noir et blanc dont l'approche formelle fait écho à ses travaux photographiques sur ce même lieu. Grâce au dispositif de tournage, léger, il gagne une vraie place au sein de l'asile, et peut interagir avec les pensionnaires. Alors que pour son film de 1974, il n'intervenait pas dans le déroulement de l'action, sa position est tout autre dans San Clemente. Il aborde son sujet en faisant de son expérience une composante à part entière du film. Il laisse les malades s'adresser à la caméra, l'interpeller, ceux-ci réagissent clairement aux moyens de la prise de vue, rappelant chaque fois qu'il s'agit bien d'une image qui établit la médiation entre le réel et le spectateur. Et que cette image est faite par quelqu'un, qui devient 
plus qu'un opérateur, en s'impliquant en tant que personnalité filmante dans la matière audiovisuelle.

Comme le dit lui-même Depardon, il n'y a rien de spectaculaire à montrer, il s'agit de la vie quotidienne, d'un temps qui se répète, de gens emportés dans une sorte d'errance : rien ne se passe vraiment. Le temps de l'expérience que constitue le tournage permet au film de se construire progressivement, dans la durée. Le « sujet » n'est plus, comme pour le journaliste, présent en tant que tel dans une réalité qui lui est extérieure. Dans la démarche participative, ce sujet naît de la rencontre entre filmeurs et filmés. Le réel documenté est composite : fait à la fois d'une situation préexistante et de sa transformation par le film en train de se faire. Il est une expérience partagée, où s'implique et s'expose le sujet-réalisateur.

Ainsi, chez Depardon, la perte de la croyance en l'objectivité photographique semble avoir contaminé le médium cinématographique. André Rouillé, plutôt que de parler d'une simple « crise du photojournalisme », décrit un phénomène plus profond : celui de la crise de l'image-document. Cette crise remet en cause la valeur de la photographie basée sur « la foi dans [sa] valeur référentielle (...) et vouant un culte au référent » (Rouillé, 2005 : 172). De là émerge ce que Rouillé nomme la «photographie-expression » qui correspond à un nouveau régime documentaire des images, pouvant être étendu au cinéma.

Pour Rouillé, la crise de l'image-document n'a fait que révéler l'utopie de la photographie comme présentation vraie du réel, en rendant visible les puissances du faux à l'œuvre dans la construction de l'image. La photographie ne peut être envisagée uniquement du point de vue du «ça-a-été » barthésien et autres théories de l'index. Ainsi, le photographe ne peut plus prétendre à montrer les choses telles qu'elles sont, la photographie n'est plus invisible. Pour Rouillé :

La photographie-document ne met pas le réel et l'image face-à-face, dans une relation binaire d'adhérence directe. Entre le réel et l'image s'interpose toujours une série infinie d'autres images, invisibles mais opérantes, qui se constituent en ordre visuel, en prescriptions iconiques, en schémas esthétiques. Le photographe n'est pas plus proche du réel que ne l'est le peintre quand il travaille devant sa toile. (Rouillé, $2005: 206$ ).

Cette idée selon laquelle la photographie n'entretient pas de rapport direct avec le réel rejoint la conception de Deleuze d'une énonciation indirecte libre comme régime du cinéma direct. Les images sont et font l'événement, elles le font exister, se développer : elles sont « la production d'un réel nouveau (...) au cours d'un processus combiné d'enregistrement et de transformation de quelque chose du réel donné, en aucun cas assimilable avec le réel » (Rouillé, 2005 : 94). 
L'image-expression a ainsi :

Une face tendue vers les choses et l'autre face vers les images. (...) elle se distingue formellement de la photographie-document et de la photographie artistique. Elle s'en distingue aussi philosophiquement. À la différence de la première, la photographie-expression ne confond pas le sens avec les choses qu'elle désigne; à l'inverse de la photographie artistique, elle ne limite pas le sens aux images et à leurs formes.

Et de là vient le sens de la démarche documentaire d'auteur :

Le sens a besoin à la fois des choses et du langage, de référents (qui « adhèrent ») et d'une écriture qui fasse déborder l'image hors des limites de l'enregistrement. Le sens survient aux choses, mais c'est l'écriture qui le retient dans ses rets. (Rouillé, 2005 : 217-218).

Avoir une face tournée vers le réel et l'autre tournée vers les images semble être également une des spécificités du type de cinéma indirect libre que pratique Depardon à partir des années 1980. Cependant, les modalités de cette implication de Depardon dans une écriture subjective diffèrent dans ses deux pratiques. À travers la photographie, Depardon se tourne assez tôt vers le registre autobiographique. Au cinéma, il faudra attendre la fin des années 1990 pour qu'il envisage également cette voie. Dans les années 1980, dans sa pratique filmique, il retrouve d'abord la question éthique de la «bonne distance » qu'il avait en photographie, qui régit la relation entre le réel et sa mise en image par l'auteur. Depardon ne prétend plus documenter le réel comme préexistant, défini, signifiant, extérieur. Sa démarche prend en compte ses choix subjectifs (sujets, modes d'intervention, aspects formels...) et semble correspondre à ce qu'Olivier Lugon qualifie de «neutralité singulière » (Lugon, 2006 : 11).

La « neutralité singulière » théorise l'aspiration documentaire qui consiste à vouloir montrer «les choses telles qu'elles sont tout en apposant à l'image sa signature » : il s'agit pour Olivier Lugon d'un "modèle privilégié de la pratique documentaire, ce que l'on pourrait appeler 'anonymat d'auteur', une esthétique de la 'neutralité singulière'», une "impersonnalité paradoxale ». Il semble que c'est ce qui caractérise les documentaires de la «maturité » de Depardon et en particulier Urgences (1987) et Délits Flagrants (1994). En effet, ces deux films sont marqués par un dispositif de tournage qui semble avant tout être au service de ce qui est filmé (caméra fixe, plans-séquences, cadrage d'ensemble), mais qui est le fruit de décisions singulières. Ces choix sont ceux de Depardon auteur, qui par ce dispositif théoriquement transparent, crée son «style » distancié, « dégageant l'écoute ».

Cette tendance à la «neutralité singulière » est avant tout une signature du regard, et apporte une force aux films documentaires de Depardon les plus connus. Elle cohabite dès le début de son ouverture journalistique avec 
une tendance plus explicite d'affirmation de soi : l'autobiographie, la signature du je. Cette dualité dans la démarche documentaire - dont les territoires sont constamment hybridés, superposés, juxtaposés par exemple dans la fiction Empty Quarter, une femme en Afrique (1985) - témoigne de l'oscillation perpétuelle de Depardon entre «parler de soi » et «parler du monde ». Depardon formule cette problématique en reprenant les concepts de «mirror » et «window » de John Szarkowki :

[La] notion de « Mirror et Window », développée dans les années soixante par John Szarkowki, recouvre l'idée selon laquelle le photographe soit se photographie lui-même, soit photographie les autres. Cette théorie est la base même de la photographie, puisqu'elle contient tout le problème du cadre. Se photographie-t-on en photographiant les gens ou est-ce qu' au contraire on est complètement «fenêtre », en photographiant les autres, en les regardant, en étant voyeur? (Depardon, 2000 : 98).

Par extension, nous pouvons affirmer que tout documentaire cinématographique est lui aussi travaillé non seulement par la réalité du référent (« un état du monde ») mais aussi par l'expérience de celui qui le saisit.

\section{De l'auteur au spectateur : autobiographie et intersubjectivité documen- taire}

En se rapprochant du champ artistique, Depardon n'est plus le reporter témoin, mu par le désir de faire circuler les informations récoltées dans le monde entier. Il se désengage d'une image conçue pour éveiller les consciences de façon forte et immédiate et abandonne sa mission de journaliste : son engagement prend alors une forme différente. Il avoue lui-même ses faiblesses comme reporter, dans Afriques, comment ça va avec la douleur ? (1996) où, à propos de son absence lors du génocide au Rwanda, il déclare :

Je sais qu'il faut du courage pour être voyeur et témoin historique, moi je suis lâche, j'ai peur, je n'aime pas cette violence évidente, mon obsession est ailleurs.

De cette vulnérabilité assumée du filmeur naît une nouvelle rencontre avec le monde. Dans ce film tourné entre 1993 et 1996, Depardon présente sa remontée de l'Afrique, du Cap au Caire, en passant par Soweto, le Karoo, Johannesburg, l'Angola des hauts plateaux, les camps de réfugiés du Rwanda et du Burundi, l'Éthiopie, la Somalie, le Soudan... Les images tournées sur place sont accompagnées de sa voix en off, relatant ses pensées et sentiments vis-àvis des « douleurs » de l'Afrique. Parfois, il explicite le contenu d'une scène filmée et son commentaire occupe la fonction de légende. À d'autres moments, il nous livre ses questionnements sur sa place de cinéaste ou d'homme occi- 
dental, au fil d'un monologue intérieur entretenant un lien fluctuant avec les images. Ses obsessions, qui dialoguent avec le monde extérieur, le conduisent à faire des images autrement : comme si l'expérience singulière de son regard rencontrait légèrement à contretemps ce qui arrivait au monde. L'association de longs plans-séquences et de la voix-je permet à cette dualité de prendre corps dans le film, créant deux fils de réalité en coprésence : l'un intériorisé, l'autre extériorisé.

Un motif récurrent témoigne du double mouvement qui est au cœur du film : de lents panoramiques circulaires à trois cent soixante degrés permettent à l'auteur de décrire l'environnement autour de lui, tout en renvoyant à sa propre position spatiale et auctoriale. Ces cercles de la caméra qui rythment le film le font apparaître comme un centre invisible qui renvoie sans cesse vers l'extérieur, en même temps qu'il se réfère à lui-même. Cette figure d'un auteur littéralement centrifuge et centripète souligne la complémentarité entre récit de soi et souci du monde à l'œuvre chez Depardon. Loin d'entériner la faillite du projet documentaire, l'émergence du je filmant permet au spectateur non pas de regarder l'auteur, mais de regarder le monde avec lui, et pourquoi pas contre lui. Loin de l'illusion d'un sens qui se présenterait de lui-même, grâce à ce dispositif réflexif, le spectateur peut participer à la fabrication active d'un sens commun. En s'affirmant comme sujet, l'auteur invite le spectateur à se faire également sujet de l'adresse et donc sujet du regard sur le monde.

Il ne faudrait pas cependant grossir le trait de l'évolution de Depardon, d'un journalisme inconscient de lui-même, à un documentaire mature, pour aboutir enfin à des œuvres à la dimension autobiographique prépondérante. S'il ne fait plus de reportages d'actualité comme dans les années 1970, il continue à s'intéresser aux institutions françaises : après le très personnel Afriques (1996), il filmera en 2003 le tribunal correctionnel de Paris dans $10^{\text {ème }}$ chambre, instants d'audience. Son parcours n'est donc pas mu par le désir de trouver une forme qui dépasse la précédente. Il s'agit plutôt d'élargir son champ de possibilités et de, chaque fois, travailler un registre en adéquation avec le sujet traité. L'autobiographie ne devient donc pas chez Depardon une nouvelle norme : il s'agirait plutôt d'une « gradation » autobiographique, clairement visible dans sa série des Profils paysans (2000, 2005, 2008).

La trilogie paysanne est née du désir de Depardon de se confronter à la réalité contemporaine du monde rural et agricole. Il s'agit d'un sujet qui concerne le monde commun : faire le constat de la disparition d'un monde d'agriculteurs indépendants. Mais il relève également d'une recherche personnelle : aller à la rencontre de personnes qui représentent le mode de vie auquel l'adolescent a renoncé en montant à Paris pour devenir photographe, et qui sont proches 
de ses parents, jamais filmés. Sa démarche de documentariste est ainsi animée d'une aspiration très privée $d^{\prime}$ ' « exorcisation » de ses obsessions, mais elle n'est pas d'abord présentée comme telle au spectateur. Ce dernier n'est pas informé de la particularité du regard de Depardon sur cet univers d'éleveurs : tout en nous montrant la réalité quotidienne de ceux qu'il filme au présent, il s'agit pour lui de se mouvoir dans son histoire personnelle.

Dans L'Approche (2000), le commentaire du cinéaste se limite à informer sur les lieux, situations et noms de chaque personnage. Puis, dans les films suivants, la temporalité se complexifie pour le spectateur : Depardon revient sur les lieux de ses précédents films, retrouvant les personnes et les fermes filmées quelques années auparavant. La rencontre a déjà eu lieu et il reviendra encore; les personnages et leurs situations ont évolué : les enfants ont grandi, les plus âgés vieilli, un homme s'est marié, le travail a changé grâce à des acquisitions matérielles... Le temps perçu dans le film n'est alors plus celui d'un supposé présent, il est intimement lié à ce que Depardon a tourné et montré avant et/ou après : la temporalité propre à chaque film s'inscrit dans un ensemble plus complexe et étiré. Intervenant d'une façon de plus en plus visible, le réalisateur fait référence à différents temps : passé ou futur de l'image, ainsi que, finalement, à son histoire personnelle. Sa relation aux personnes filmées évolue d'une façon visible à l'écran : il s'adresse à eux sans plus chercher à dissimuler sa parole dans La Vie moderne (2008) - «- Tu te souviens quand je t'ai filmé dans la grange là ? - Oui, je me souviens, c'était il y a deux ans, beaucoup de choses ont changé depuis... ». Depardon assume cette fois la dimension autobiographique forte qui l'a conduit à se rendre sur ces lieux, et à faire part à son spectateur de ce qu'il cherchait. L'ensemble temporel que constituent les trois films en est transformé. Au fil de la trilogie, l'auteur rend discrètement sensible l'histoire qui le rattache affectivement à ceux qu'ils filment.

En filmant le présent d'une transformation sociale, c'est donc aussi sa mémoire qu'il met en image. Depardon est fils de paysan et le lieu de son enfance : la ferme du Garet, quittée à ses seize ans, fait figure pour lui de lieu originel. Dans son œuvre, il s'agit souvent de chercher un équivalent à ce lieu, soit en se réappropriant la ferme, en la réinvestissant de sa mémoire dans son livre La Ferme du Garet, soit en allant à la recherche d'alter ego, en Afrique ou en France, à travers sa série des Profils paysans. Certains pourraient conclure à un « égotisme » photographique ou cinématographique, notion souvent attachée à une frange de l'art contemporain, dans sa tendance à un intérêt pour l'intime et le banal, prenant l'auteur et sa vie quotidienne comme sujets privilégiés (Poivert, 2002). En effet, le souci critique de s'exposer soi-même risque sans cesse se retourner en une stratégie de distinction narcissique : dire je serait aussi 
l'expression du désir voire de la prétention d'être reconnu comme auteur, précédant le désir de parler du et depuis le monde commun. Cependant, Depardon est loin de cet art de l'intime et n'abandonne jamais l'attention à l'autre et le souci du monde qui caractérise la tradition documentaire.

En cela, sa trajectoire correspond à l'évolution historique du rapport à la photographie documentaire : d'abord nécessairement ancrée dans le réel qu'elle enregistre, sa valeur de vérité est mise en doute lors d'une «crise de l'image-document ». Les photographes glissent alors vers des pratiques plus explicitement subjectives. Si nombre de penseurs attachés à l'art comme objet autonome aiment à considérer ce changement comme une forme de libération, il nous semble plus pertinent d'y voir une forme de complexification. Car l'image-expression ne vise pas à se libérer du monde dans lequel nous vivons, mais plutôt à y faire référence de façon enrichie, qui soit fidèle à la manière dont nous donnons sens au réel : par la rencontre et la transformation réciproque entre ce qu'il y a «out there » et ce que nous portons « in there».

En abandonnant la recherche de l'objectivité vis-à-vis du monde extérieur, le cinéaste documentariste ne renonce pas à tout sens partageable pour livrer une vision du monde repliée sur lui-même. Au contraire, son je s'adresse à nous spectateurs, de manière plus humble et plus directe. Ainsi, plutôt que de distinguer les registres de l'objectivité et de la subjectivité, il apparaît en fait que le registre du documentaire d'auteur est celui de l'intersubjectivité. C'est grâce à ce concept que Jean-Luc Lioult propose de dépasser pragmatiquement la question récurrente de l'impossible objectivité du documentaire. Il définit l'intersubjectivité comme « la capacité sociale qu'ont des sujets à se mettre d'accord sur un jugement concernant l'état du monde » (Lioult, 2010). Dans le cas du documentaire, la validité d'une représentation ne proviendrait plus de règles internes au discours mais plutôt de la rencontre des regards de l'auteur et du spectateur. Ces derniers peuvent s'accorder sur une vision commune de la réalité : le consensus intersubjectif permet de rendre partageable une certaine vision du monde, même si elle se fait à partir de l'expérience proposée par le documentariste.

Plus qu'une contradiction entre le mouvement d'objectivation documentaire et celui de subjectivation autobiographique, l'autobiographie documentaire fait apparaître la dimension intersubjective de tout film documentaire. La porosité entre des formes plus ou moins autobiographiques chez Depardon nous prouve que l'impulsion de dire je, loin d'entrer en concurrence avec le projet documentaire, en est plutôt le parangon. Elle rend explicite la nature nécessairement intersubjective de «l'état du monde » dont nous parle le cinéma. 


\section{Références bibliographiques}

Barthes, R. (1980). La chambre claire, notes sur la photographie. Paris: Ed. Cahiers du Cinéma, Gallimard Seuil.

Calder-Marshall, A. (1963). The innocent eye, the life of Robert Flaherty. New York: Harcourt, Brace \& World.

Cartier-Bresson, H. (1952). L'instant décisif. Images à la sauvette. Paris: Verve, np.

Deleuze, G. (1985). Cinéma II: l'image-temps. Paris: Ed. de Minuit.

Deligny, A. (2004). Viva, une alternative à Magnum?. Études photographiques, (15): 78-103.

Depardon, R. (2006). Image, voyage, vingt ans après. Entretien avec JeanFrançois Chevrier [1998]. La Solitude heureuse du voyageur, précédé de Notes: 79-106. Paris: Ed. Seuil.

Depardon, R. (2006). Introduction. La solitude heureuse du voyageur, précédé de notes: 8-19. Paris: Ed. Seuil.

Depardon, R. (2007). L'être photographe, entretiens avec Christian Caujolle. Paris: Ed. de l'Aube.

Freund, G. (1974). Photographie et société. Paris: Ed. Points Seuil.

Lioult, J.-L. (2004). À l'enseigne du réel, penser le documentaire. Aix-enProvence: Université de Provence.

Lioult, J.-L. (2010). Théorie du film documentaire (Penser le cinéma documentaire: leçon 3). Disponible sur : http://www.canalu.tv/video/tc p_universite_de_provence/theorie_du_film_documentaire_penser_le_cin ema_documentaire_lecon_3.6784

Lugon, O. (2006). L'anonymat d'auteur. Document 3, Le statut de l'auteur dans l'image documentaire: signature du neutre: 6-14. Paris: Ed. du Jeu de Paume.

Nichols, B. (2001). Introduction to documentary. Bloomington, Indianapolis: Indiana University Press.

Parente, A. (2005). Cinéma et narrativité. Paris: L’Harmattan.

Pierrmon-Moinel, M.-J. (2010). Modernité et documentaires: une mise en cause de la représentation. Paris: L'Harmattan.

Poivert, M. (2002). La photographie contemporaine. Paris: Flammarion.

Rancière, J. (2000). L'art de la distance. In R. Depardon, Détours. Paris: Ed. Maison Européenne de la Photographie, np. 
Rouillé, A. (2005). La photographie, entre document et art contemporain. Paris: Gallimard.

Roussin, P. (2006). Quelques remarques à propos de l'auteur, du documentaire et du document. Document 3, Le statut de l'auteur dans l'image documentaire: signature du neutre: 34-49. Paris: Ed. du Jeu de Paume.

Saussier, G. \& Chérel, E. (2006). La place de l'auteur et du spectateur dans la photographie documentaire. Document 3, Le statut de l'auteur dans l'image documentaire: signature du neutre: 14-24. Paris: Ed. du Jeu de Paume.

Schaeffer, J.-M. (1999). Pourquoi la fiction? Paris: Seuil.

\section{Filmographie}

Nanouk, l'Esquimau (1922) de Robert Flaherty

Primary (1960) de Robert Drew et Richard Leacock

Chronique d'un été (1961) de Jean Rouch et Edgar Morin

Jan Palach (1969) de Raymond Depardon

Tchad 1: l'Embuscade (1970) de Raymond Depardon

1974, Une Partie de campagne (1974/2002) de Raymond Depardon

San Clemente (1980) de Raymond Depardon

Urgences (1987) de Raymond Depardon

Délits flagrants (1994) de Raymond Depardon

Afriques, Comment ça va avec la douleur ? (1996) de Raymond Depardon

Série des Profils Paysans : L'Approche (2000), Le Quotidien (2005), La Vie moderne (2008) de Raymond Depardon

\section{Recueils de photographies}

Depardon, R. (1979), Notes, Paris: Arfuyen.

Depardon, R. (2006), Correspondance New-Yorkaise [1981], In New York, Paris: Ed. des Cahiers du Cinéma.

Depardon, R. (1995), La Ferme du garet, Paris: Carré.

Depardon, R. (1998), La solitude heureuse du voyageur, Paris: Seuil.

Depardon, R. (2000), Errance, Paris: Seuil.

Depardon, R. (2000), Détours, Paris: Maison Européenne de la Photographie.

Frank, R. (1958), Les Américains, Paris: Delpire. 